\title{
Géolinguistique
}

15 | 2015

La géographie linguistique au Brésil

\section{Atlas Lingüístico do Paraná}

Linguistic Atlas of Paraná

\section{Vanderci de Andrade Aguilera}

\section{(2) OpenEdition}

Journals

Édition électronique

URL : http://journals.openedition.org/geolinguistique/631

DOI : 10.4000/geolinguistique.631

ISSN : 2650-8176

\section{Éditeur}

UGA Éditions/Université Grenoble Alpes

\section{Édition imprimée}

Date de publication : 1 décembre 2015

Pagination : 101-119

ISBN : 978-2-84310-317-9

ISSN : 0761-9081

\section{Référence électronique}

Vanderci de Andrade Aguilera, "Atlas Lingüístico do Paraná », Géolinguistique [En ligne], 15 | 2015, mis en ligne le 15 février 2019, consulté le 02 novembre 2020. URL : http://journals.openedition.org/ geolinguistique/631 ; DOI : https://doi.org/10.4000/geolinguistique.631 


\title{
Atlas Lingüístico do Paraná
}

\author{
Vanderci de Andrade Aguilera \\ Universidade Estadual de Londrina (Brésil)
}

\section{Résumé}

L'Atlas Lingüístico do Paraná - ALPR (Aguilera, 1994) est le résultat de la thèse de doctorat soutenue en 1990. Il s'agit du cinquième atlas régional qui a vu la lumière après l'Atlas Prévio dos Falares Baianos - APFB (Rossi, 1963), l'Esboço de um atlas Lingüístico de Minas Gerais - EALMG (Ribeiro et al., 1977), l'Atlas Lingüístico da Paraíba - ALPB (Aragão \& Menezes, 1984) et l'Atlas Lingüístico de Sergipe - ALS (Ferreira, 1987). L'ALPR repose sur des enquêtes de terrain et un réseau de 65 points, avec deux informateurs par localité, un homme et une femme issus du milieu rural ou résidents à la campagne, d'un âge compris entre 30 et 50 ans, préférentiellement analphabètes ou peu scolarisés. Les informateurs ont répondu à des questionnaires de nature phonétique et lexical, pour un total de 325 questions posées sur deux domaines principaux : l'Homme et la Terre. L'ALPR est composé de 191 cartes, dont 92 lexicales, 70 phonétiques et 29 d'isoglosses. On sait que les limites des dialectes ne sont pas fixes, mais qu'ils varient en fonction de différents facteurs. Cependant, la photographie linguistique de l'Atlas Lingüístico do Paraná peut classer les parlers en deux groupes principaux : (i) parler du Nord et du Sud et (ii) parler du Paraná traditionnel et du Paraná moderne. Pour distribuer les dialectes du Nord et du Sud, on a saisi comme base, entre autres faits phonétiques, la distribution spatiale $\mathrm{du} / \mathrm{r} /$ en position d'attaque : dans le Nord s'impose la consonne /r/ glottale (voire vélaire) et la distribution des voyelles atones finales /e/ et /o/ dans cette région-là sont généralement élevées. Dans le parler du Sud, le /r/ est surtout alvéolaire multiple, lorsque les voyelles moyennes finales restent sans contrainte. Le classement des dialectes en traditionnel et moderne est basé sur des isoglosses de certains éléments lexicaux tels que corvo et urubu, córrego et arroio/sanga, cuitelo et beija-flor, maçanilha et camomila, entre autres. 


\title{
Mots-clés
}

Paraná, atlas linguistique, Brésil.

\begin{abstract}
The Linguistic Atlas of Paraná (Atlas Lingüístico do Paraná - ALPR) (Aguilera, 1994) is the outcome of a PhD thesis finished in 1990. It is the fifth state atlas which came to light after the publication of the Atlas of the Speech in Bahia (Atlas Prévio dos Falares Baianos - APFB) (Rossi, 1963), the Draft of a linguistic atlas of Minas Gerais (Esboço de um atlas Lingüístico de Minas Gerais - EALMG) (Ribeiro et al., 1977), the Linguistic Atlas of Paraíba (Atlas Lingüístico da Paraíba-ALPB) (Aragão \& Menezes, 1984) and the Linguistic Atlas of Sergipe (Atlas Lingüístico de Sergipe - ALS) (Ferreira, 1987). To carry out the ALPR, a 65-point network was established, and in each point a man and a woman, ranging from 30 to 65 years of age, preferably illiterate or with low literacy level, of rural origin or living in the countryside, were interviewed. The field research consisted in the application of a questionnaire with 325 questions about phonetic and lexical aspects, on two big fields: Man and Earth. The ALPR comprises 191 letters, out of which 92 are lexical, 70 are phonetic and 29 are isoglossal. It is known that the dialectal limits are not fixed, but variable according to a variety of factors. However, in the linguistic picture the atlas presents, the speech in Paraná can be classified in two big groups: (i) the speech from the North and South and (ii) the speech from the Traditional Paraná and the Modern Paraná. In order to distribute the Northern and the Southern speech, among other phonetic factors, the atlas took as a basis the areal distribution of $/ r \mid$ in onset position which, in the North, predominates as velar, and the distribution of the final voiceless middle vowels /el and /o/ which, in that region, are normally raised. In the Southern speech, the $|r|$ is predominantly alveolar and multiple and the final voiceless middle vowels remain. To classify the speech in traditional and modern, the isoglosses of some lexical items were documented, such as corvo and urubu, córrego and arroio/sanga, cuitelo and beija-flor, maçanilha and camomila, among others.
\end{abstract}

\section{Keywords}

Paraná, linguistic atlas, Brazil.

L'Atlas Lingüístico do Paraná - ALPR (Aguilera, 1994) est le cinquième atlas d'État publié au Brésil après la parution de l'Atlas Prévio dos Falares Baianos - APFB (Rossi, 1963), l'Esboço de um atlas Lingüístico de Minas 
Gerais - EALMG (Ribeiro et al., 1977), l'Atlas Lingüístico da Paraíba ALPB (Aragão \& Menezes, 1984) et l'Atlas Lingüistico de Sergipe - ALS (Ferreira et al., 1987). Les quatre atlas antérieurs à l'ALPR sont le résultat des efforts d'équipes de chercheurs participants à un projet institutionnel, accueilli par une université fédérale et en tant que coordinateurs, membres du corps professoral de cours de langues. L'ALPR, au contraire, est le résultat d'un projet individuel, la thèse de doctorat, développée dans le cadre du Programa de Pós-Graduação en Letras de la Universidade Estadual Paulista (Campus de Assis) de 1987 à 1990. Le sujet a été suggéré par le professeur Pedro Caruso, membre du Département de dialectologie de l'institution citée, et la thèse a été dirigée par le professeur Raphael Hoyos-Andrade.

Pour la préparation de l'ALPR, j'ai recouru tout d'abord aux atlas précédents afin d'adopter une méthodologie commune et pouvoir ainsi comparer les résultats. Cependant, les quatre atlas ont adopté des approches méthodologiques différentes : j'ai donc décidé de suivre les propos du projet de l'Atlas Lingüístico do Estado de São Paulo, coordonné par le professeur Caruso.

Une raison particulière a déterminé mon choix : Paraná et São Paulo sont des États voisins qui ont partagé la même histoire de colonisation. Le premier est resté un quartier de São Paulo depuis la fondation des premiers peuplements à la fin du XVII ${ }^{\mathrm{e}}$ siècle jusqu'au milieu du XIX ${ }^{\mathrm{e}}$ siècle, quand le Paraná a conquis son émancipation. Compte tenu de cette déclaration, je me suis posé les questions suivantes : dans quelle mesure les parlers de São Paulo et du Paraná se ressemblent-ils? En quoi sont-ils différents ? Les réponses, en l'absence d'un atlas de l'État de São Paulo, pourront être traitées par l'Atlas Lingüístico do Brasil, lorsque seront publiées les cartes qui décrivent les points de l'intérieur du pays.

\section{Localisation du Paraná dans le temps et l'espace : un aperçu historique}

Le Paraná, dont la capitale est Curitiba, est un des 26 États du Brésil, il est situé dans la région Sud. Son territoire s'étend sur $199709 \mathrm{~km}^{2}$, avec une population d'environ 11 millions d'habitants, selon le dernier recensement (IBGE, 2014).

La plupart de l'actuel territoire de l'État du Paraná appartenait à l'Espagne d'après le traité de Tordesillas signé entre le Portugal et l'Espagne en 1494. L'Espagne a fondé les colonies de Ontiveros et de Villa Rica do Espírito Santo, au milieu du $\mathrm{XVI}^{\mathrm{e}}$ siècle, à l'Ouest, tous près de la rivière 
Paraná. La côte atlantique du Paraná, par ce même traité, appartenait au Portugal et, des années plus tard, elle a été rattachée à la province de São Vicente.

Les nouveaux territoires découverts ont attirés des explorateurs européens qui cherchaient du bois, des pierres et des métaux précieux. Pour la recherche et l'extraction de ces produits, ils ont exploité le travail des Indiens autochtones. D'autre part, l'Église a travaillé avec ses missionnaires pour convertir les Indiens à la foi chrétienne. Ce fait a provoqué d'intenses conflits entre les missionnaires espagnols et les bandeirantes, descendants des portugais et des autochtones qui se sont organisés en groupes, dans le but de découvrir les mines d'or et de pierres précieuses et de capturer les Indiens pour les forcer aux travaux manuels. La progression des bandeirantes vers l'interieur, au-delà des limites fixées par le traité de Tordesillas, a donné au Brésil une conformation très proche de celle d'aujourd'hui.

Jusqu'au début de la colonisation du territoire brésilien, au milieu du XVII ${ }^{\mathrm{e}}$ siècle, commence aussi l'occupation du territoire actuel du Paraná avec la fondation de la ville côtière de Paranaguá, par des jésuites espagnols. Curitiba a été fondée à la suite et elle est élevée au rang de ville en 1693.

La vie économique de la Quinta Comarca de São Paulo, entre le XVII et le $\mathrm{XIX}^{\mathrm{e}}$ siècle, comprennait : (i) la recherche de l'or à Paranaguá, attirant les Portugais et leurs descendants indigènes (Mamelucos), non seulement vers la Côte, mais aussi vers l'intérieur; (ii) l'élevage de bœufs, pour alimenter la région de Minas Gerais, et de chevaux, des ânes et des mulets, pour le travail dans les mines. À cette époque a été ouverte la route de Viamão, que les gardiens de troupeaux parcourent pour aller de cette ville du Rio Grande do Sul jusqu'au village de Sorocaba dans l'État de São Paulo (fig. 1), où le gros bétail était vendu dans les foires agricoles. Par conséquent, le long du chemin, ont été crées des logis pour le repos des troupes et des gardiens qui, par la suite, ont été remplacés par des villages et des villes, comme Guaratuba, Antonina, Morretes, Iapó (actuellement Castro), Vila Nova do Príncipe (aujourd'hui Lapa), São José et Guaraqueçaba; (iii) l'extraction de l'herbe maté, le produit principal de l'État, depuis les premières années de la colonisation jusqu'à la fin du XIX ${ }^{\mathrm{e}}$ siècle; et enfin, (iv) la production de café et l'exploitation du bois, ont contribué à l'augmentation de la population du Nord, du Nord-Est et du Sud-Ouest du Paraná.

La figure 1 montre les chemins parcourus par les gardiens de troupeaux itinérants pendant les $\mathrm{XVII}^{\mathrm{e}}$ et XVIII ${ }^{\mathrm{e}}$ siècles, dont les logis ont donné lieu à la plupart des villes situées le long de ces routes. Tout en servant d'arrêt 
pour les hommes et les animaux, ces noyaux représentaient l'environnement idéal où les contacts linguistiques se matérialisaient et les parlers se mêlaient.

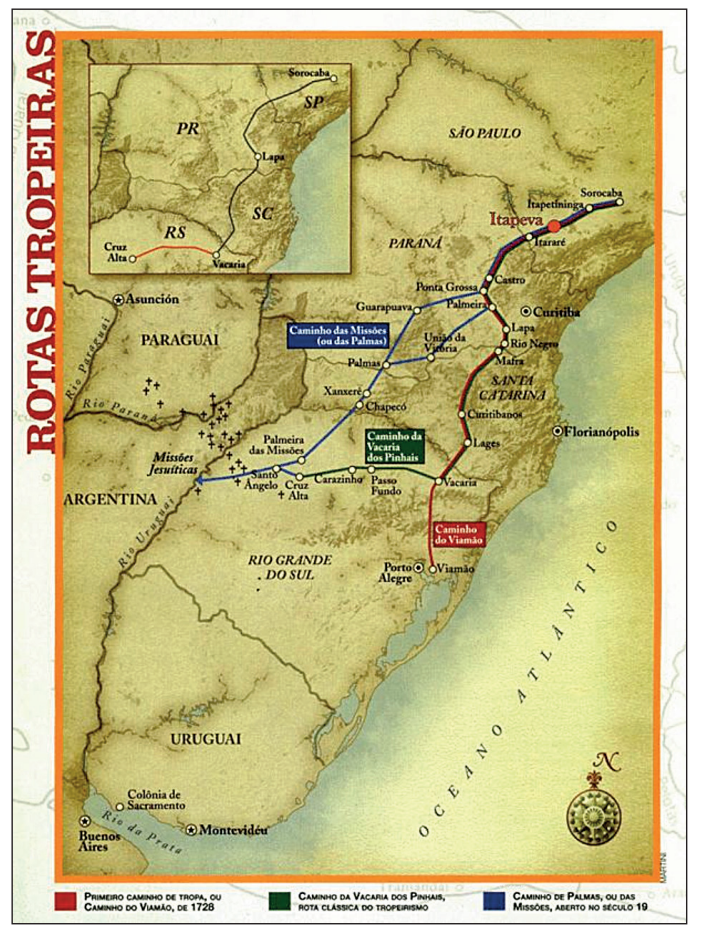

Figure 1. - Chemins de gardiens de troupeaux itinérants aux XVIII' et XIX siècles reliant le Rio Grande do Sul (Viamão) à São Paulo (Sorocaba).

Source : <www.google.com.br/search?q=Mapa+das+rotas+tropeiras+no+sul+do+Brasil> [dernier accès le 23 avril 2015].

En 1853, après la séparation de la province de São Paulo, commence l'histoire officielle de la Quinta Comarca qui s'est formée comme une province en 1859. À l'époque, le programme d'immigration européenne a fait venir des Allemands, des Polonais, des Ukrainiens et des Italiens, et pour favoriser l'économie, ont été crées les premiers chemins de fer, facilitant ainsi une meilleure intégration entre l'État de Paraná et l'État de São Paulo. Ces deux facteurs ont joué un rôle important dans la caractérisation progressive des différents dialectes du Paraná.

L'histoire de Paraná, selon Cardoso et Westphalen (1986, p. 9-11), comprend la formation de trois communautés régionales : celle du Paraná Tradicional, qui commence à prendre corps au XVII ${ }^{\mathrm{e}}$ siècle, avec la demande croissante de l'or et qui s'est structurée au cours du même siècle avec 
l'élevage et le commerce de bétail. En même temps, se sont développés tout d'abord l'exploitation de l'herbe mate, le commerce et l'exportation du bois; deuxièmement, au $\mathrm{xx}^{\mathrm{e}}$ siècle, déjà dans le Paraná Moderno, dans la région Nord s'est développée la culture tropicale du café qui, à cause des origines et des interêts historiques, était liée directement à São Paulo; troisièmement, dans le Sud-Ouest et l'Ouest du même État, ont été développé l'élévage de porcs et la production céréalière, qui la lient plus historiquement au Rio Grande do Sul.

L'occupation progressive de l'État du Paraná est un facteur à prendre en considération lorsque l'on étudie ses différents dialectes. La Côte, le Centre et le Sud ont été occupés successivement par les découvreurs puis par les immigrants européens, depuis plus de 200 ans ; plus tard, à l'aube du $\mathrm{xx}^{\mathrm{e}}$ siècle, le Nord, le Nord-Ouest, l'Ouest et le Sud-Ouest ont été occupés uniquement par les étrangers. La figure 2 montre, en couleurs, les points d'enquête de l'ALPR relatifs aux localités fondées entre les années 1900 et 1960, constituant le Paraná Moderno et, en blanc, l'espace occupé par des localités du Paraná Tradicional, fondées au cours des XVII ${ }^{\mathrm{e}}$, XVIII et $\mathrm{XIX}^{\mathrm{e}}$ siècles.

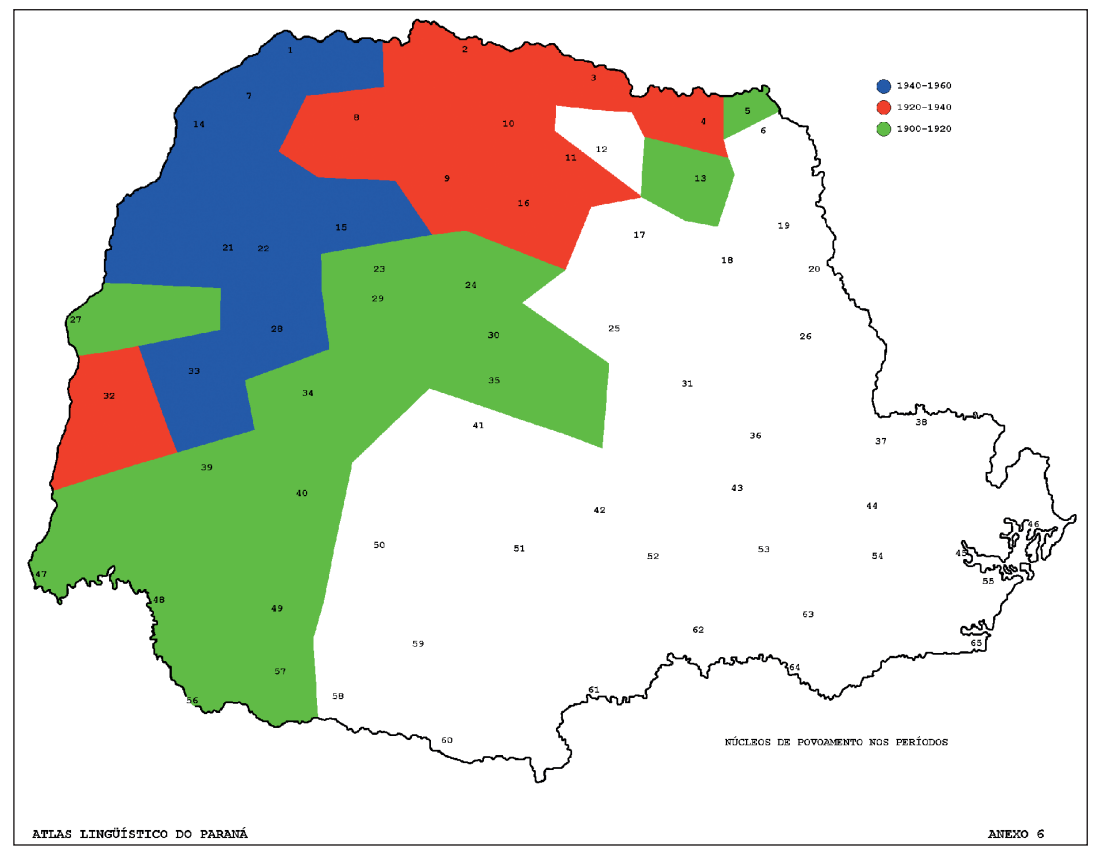

Figure 2. - Localités du Paraná Moderno (en couleurs) et du Paraná Tradicional (en blanc).

Source : Atlas Lingüístico do Paraná (Aguilera, 1994), d'après Cardoso et Westphalen (1986, p. 11). 


\section{Atlas Lingüístico do Paraná (ALPR) : génèse et approches méthodologiques}

En 1985, pendant le cours de master, en faisant l'inventaire des publications sur la diversité linguistique au Paraná, je n'ai trouvé que des études consacrées à la langue parlée dans le Sud de l'État et, en particulier, sur la Côte et au Centre. Je voudrais signaler deux ouvres : premièrement, l'article de Andrade Muricy présenté au $1^{\text {er }}$ Congresso Nacional de Língua Falada, qui s'est tenu à São Paulo en 1938, sous la rubrique «Algumas vozes regionais do Extremo Oeste do Paraná». L'auteur présentait une liste d'un peu plus de deux cents mots, tirés de notes écrites par son père, le général José Cândido Muricy, lors d'un voyage d'exploration à l'intérieur du Paraná et, en particulier, dans le Centre et le Sud-Ouest de l'État. Muricy enregistrait les mots les plus significatifs et les expressions utilisées par les personnes avec lesquelles il avait eu des contacts pendant le voyage pour se rendre à l'endroit où il supposait l'existence de la Villa Rica dos Jesuítas, fondée au XVI ${ }^{\mathrm{e}}$ siècle (Muricy, 1975). Deuxièmement la thèse de doctorat de José Luiz da Veiga Mercer, Le lexique technique des pêcheurs de Guaraqueçaba (1979), qui fournit une liste exhaustive et méticuleuse du vocabulaire de la pêche côtière. Dans le cadre du programme du master, a été présentée la proposition de Pedro Caruso, auteur du projet Atlas Lingüístico do Estado de São Paulo (1983), d'entreprendre un Atlas Lingüístico do Paraná dans la mesure où peu d'études avaient été consacrées aux dialectes de cet État, proposition qui sera à l'origine de ma thèse de doctorat. Par la suite je constatais que dans quelques universités fédérales il y avait déjà un projet pour l'élaboration d'un atlas de la région Sud, englobant trois États : Santa Catarina, Paraná et Rio Grande do Sul. Puisque ma thèse était déjà en cours et que, par certains aspects, elle différait dans sa conception, j'ai continué mes recherches en considérant que l'existence de deux atlas ne pouvait qu'enrichir la connaissance des variétés dialectales de cette région.

\section{Le questionnaire et les enquêtes}

Pour le recueil des données, j'ai opté pour la recherche directe, avec des enquêtes structurées, plutôt que celle indirecte (par exemple avec des questionnaires envoyés par correspondance). Après les suggestions des questionnaires de Silva Neto (1957), Nascentes (1958 et 1961) et de Caruso (1983), j'ai choisi le questionnaire de l'Atlas do Estado de São Paulo (ALESP) parce que les données des uns et des autres pourraient être facilement comparées plus tard. Une autre raison a pesé dans ce choix, à savoir le facteur 
historique, l'occupation de l'État du Paraná, pendant près de quatre siècles, se confondant avec celle de São Paulo.

Ce questionnaire avait également été testé à Cascavel et à São Mateus do Sul, villes situées respectivement à l'Ouest et au Sud, et il a été utilisé dans les douze communes du réseau de l'Atlas linguistique de Londrina (EALLO) - mon diplôme de master (Aguilera, 1987).

Après quelques mises au point, le questionnaire, comportant 318 questions, directes et indirectes, réparties sur deux grands champs sémantiques : l'Homme et la Terre qui, à son tour, impliquent des sous-champs : la nature (57 questions), la flore (37); les plantes médicinales (15); la faune (50); le corps et les maladies (105); l'habillement (14); l'agriculture et les outils agricoles (29); les jouets et les jeux (13). Au questionnaire, s'ajoutent cinq récits sur des traits culturels régionaux, des croyances populaires et des expériences personnelles : le corpus enregistré répond, au total, à 325 questions. Les enregistrements ont été transcrits et imprimés : ils sont rassemblés dans 13 volumes, inédits, comportant environ 2000 pages.

Par rapport au questionnaire de l'ALESP, beaucoup de modifications ont été introduites dans celui de l'ALPR en tenant compte des suggestions de nos informateurs : par exemple, j'ai ajouté l'entrée camomille qui ne figurait pas dans le questionnaire de l'ALESP, dans la mesure où j'ai trouvé qu'un informateur de São Mateus do Sul donnait la désignation maçania qui est une variante régionale du sud du Brésil pour cette plante. Certaines questions, révèlent l'existence de tabous linguistiques : par exemple, pour désigner une chute de grêle, on évite le mot pierre, considéré comme offensif à l'égard de Dieu, le Créateur, et on le remplace par pluie de fleurs, pluie de roses ou pluie de blé. Toujours dans ce domaine, d'autres noms, se référant au corps et à l'habillement féminins, comme l'utérus, les seins, l'avortement et le soutien-gorge ont été prononcé, comme dans les manifestations de timidité ou de honte, avec une intensité plus faible de la voix par rapport à la normale. Encore un exemple : pour nommer un pont rudimentaire, généralement appelé pinguela, des informateurs de certaines localités ont évité d'employer ce nom car, dans leur région, le mot désigne le sexe masculin.

Le questionnaire de ALESP comporte des questions ouvertes concernant par exemple les types ou les variétés de poules, de haricots, de riz, de cafés, de citrons ou de papayes, connus dans les localités. Faute d'emploi de génériques pour ces référents, il était difficile de cartographier les réponses, chaque locuteur répondant par rapport à ses propres connaissances : sans compter que, lors de l'enquête, il avait tendance à employer le premier mot qui venait à sa mémoire. Par conséquent j'ai inclu des questions plus précises pour mieux diriger l'informateur. Ainsi, par exemple, 
la question 87, concernant la poule, a été subdivisée de façon à obtenir les caractéristiques des espèces nommées, comme caipiras, garnisé, arrepiada, pescoço pelado, ligórnia, carijó, índia, entre autres (il s'agit de désignations locales de différentes espèces de poules).

Le recueil de données à partir de 130 informateurs a été réalisé par un petit nombre d'enquêteurs : l'auteur (74\% des enquêtes), aidée par ma collègue Ivone Lima et par quelques étudiants de lettres de l'Universidade Estadual de Londrina. Pendant six ans (1985-1990), nous avons couvert environ $50000 \mathrm{~km}$ à l'intérieur du Paraná, en enquêtant dans 65 points, appartenant à une centaine de communautés rurales ou péri-urbaines.

\subsection{Le nombre et les caractéristiques des points d'enquête}

Au moment de la planification du projet de l'ALPR, le Paraná comptait 323 localités. Pour que l'échantillon des dialectes soit significatif, nous étions d'avis que les points d'enquête devaient impliquer toutes les régions géographiques, comportant les localités anciennes et récentes, et situées à une distance d'environ $50 \mathrm{~km}$ l'une de l'autre. Au début, nous avons pris en compte 24 localités suggérées par Nascentes (1958) mais, par la suite, étant donné qu'elles étaient concentrées dans le Sud et sur la Côte, nous avons étendu leur nombre à 50 , pour atteindre les 65 points, couvrant toutes les régions, à la fin de la recherche.

\subsection{Les informateurs}

Il est généralement admis que le succès d'une recherche dialectologique réside surtout dans le bon choix des informateurs et des stratégies appropriées pour les enquêtes. Au début, nous avons choisi d'interroger systématiquement deux informateurs par point d'enquête, un homme et une femme, en prévision d'une analyse de la variation diasexuelle, aboutissant à un atlas bidimensionnel, contrairement aux atlas précédents qui étaient unidimensionnels. Pour les autres critères, nous avons suivi les principes de la dialectologie traditionelle préconisant que la fourchette d'âge des parlants devaient être de 30 à 55 ans, d'origine rurale, être nés dans la localité de l'enquête, être analphabètes ou avoir un niveau de scolarité très bas.

À première vue, il nous semblait facile de trouver à la campagne deux personnes avec ce profil. L'expérience, cependant, a montré que nous ne pouvions pas être trop exigeants et que nous devions établir des critères moins rigides. Par exemple, dans les villes de moins de 50 ans ou fortement industrialisées, dans l'impossibilité de trouver un informateur analphabète nous devions choisir entre un individu instruit mais originaire de la localité et un analphabète provenant de la région d'origine de ses fondateurs. Un autre critère était de chercher des informateurs appartenant à différentes 
familles, en évitant d'interroger un couple, pour relever des variations éventuelles. Dans quelques cas, nous avons dû renoncer à ce critère.

Dans la dialectologie traditionnelle on exigeait aussi que l'informateur possède une bonne dentition et qu'il comprenne le sens des questions. Le premier critère s'est révélé improbable dans les villages de l'intérieur; le deuxième, irréalisable au premier contact, outre le fait de représenter un critère subjectif. Pour le premier critère nous avons vérifié que le locuteur pronnonçait bien des constrictives comme /f/, /v/, /s/ et /z/. Au début des entretiens il fallait aussi vérifier l'interêt de l'informateur à collaborer à notre enquête. Enfin, nous avons pu dresser le tableau suivant concenant les informateurs :

\begin{tabular}{|c|c|c|c|c|}
\hline Groupe d'âge & homme & femme & total & $\%$ \\
\hline $27-30$ & 4 & 2 & 6 & 4,6 \\
\hline $31-40$ & 25 & 21 & 46 & 35,4 \\
\hline $41-50$ & 22 & 28 & 50 & 38,5 \\
\hline $51-62$ & 14 & 14 & 28 & 21,5 \\
\hline Total & 65 & 65 & 130 & 100,0 \\
\hline
\end{tabular}

Tableau 1. - Profil des locuteurs, par sexe et classes d'âge.

On observe que la majorité des locuteurs, soit $74 \%$, se situe entre 30 et 50 ans, une catégorie de personnes ni trop jeunes, ni trop vieilles.

\begin{tabular}{|c|c|c|c|c|}
\hline Niveau d'education & homme & femme & total & $\%$ \\
\hline Analphabète & 39 & 38 & 77 & 59,3 \\
\hline éducation de jeunes adultes & 7 & 6 & 13 & 10,0 \\
\hline $1^{\text {er }}$ degré incomplet & 15 & 21 & 36 & 27,7 \\
\hline $1^{\text {er }}$ degré complet & 4 & - & 4 & 3,0 \\
\hline Total & 65 & 65 & 130 & 100,0 \\
\hline
\end{tabular}

Tableau 2. - Profil des répondants selon le sexe et le niveau d'éducation.

La plupart des informateurs présentent un faible niveau d'instruction (97\% ont moins de quatre années d'études). Étant donné que leur profil est plutôt homogène, cela permet une analyse diatopique avec peu d'influences des facteurs diastratiques ou sociaux.

\section{La préparation des cartes linguistiques}

Pour la préparation des cartes, nous avons dû créer une méthodologie propre, aucun des quatre atlas publiés précédemment n'ayant présenté clairement les procédures méthodologiques employées. L'expérience de l'EALLO (Aguilera, 1987) a grandement facilité la tâche. Par ailleurs, 
l'Atlas Lingüístico de Sergipe (Ferreira et al., 1987), publié quand nous avons commencé les enquêtes de l'ALPR, montrait déjà un certain nombre de progrès par rapport aux précédents, comme l'indication du sexe des informateurs indiqué sur les cartes $\mathrm{A}$ et $\mathrm{B}$ à côté des variantes. Dans l'ALPR, nous avons choisi d'indiquer le sexe avec les symboles de la biologie (O)

Pour les cartes lexicales, nous suivons l'ordre séquentiel du questionnaire qui, à son tour, a été organisé par champs sémantiques. Ainsi, les cartes 1 à 92 montrent des variantes lexicales des champs sémantiques concernant la nature, l'homme, la géomorphologie et jusqu'aux jouets et aux jeux d'enfants.

Pour mettre en évidence la variation phonétique qui caractérise les parlers du Paraná, il n'existait pas un questionnaire spécifique. Pour répondre à ce besoin, nous avons établi des critères permettant d'enregistrer des voyelles, des consonnes et des groupes phoniques dans plusieurs contextes. Ainsi, nous avons des cartes qui présentent la variation des proparoxytons, $\mathrm{du} / \mathrm{r} /$ en position d'attaque et de coda, des diphtongues orales et nasales, $\mathrm{du} / \mathrm{l} / \mathrm{dans}$ les groupes consonantiques, des voyelles /e/ et /o/ atones finales, parmi d'autres, dans le discours populaire et/ou rural, dans les 65 points linguistiques étudiés. Pour compléter l'ensemble des cartes, nous avons synthétisé les données de dix-neuf cartes lexicales et de dix cartes phonétiques d'isoglosses permettant une visualisation plus rapide de la distribution spatiale de ces variantes.

La figure 3 montre la distribution diatopique des variantes pour la désignation du référent sol humide, à la campagne, où les gens peuvent enfoncer leurs pieds. La même figure montre que les frontières dialectales ne sont pas hermétiques, mais perméables, les deux variantes majeures, banhado et brejo, se croisant. En observant l'aire d'extension de brejo, nous constatons que cette variante se retrouve dans le parler du Norte paranaense et de la Côte, se prolongeant vers l'Ouest. Deux faits historiques du peuplement du Paraná justifient cette répartition géographique : (i) sur la Côte, cette variante est venue peut-être avec les premiers colons européens, des Portugais et leurs descendants nés au Brésil, fondateurs du village de São Vicente; (ii) le deuxième fait concerne la colonisation récente du Norte paranaense par le nombre élevé de cultivateurs de café arrivant de l'intérieur de Minas Gerais et de São Paulo installés à la fin du $\mathrm{XIX}^{\mathrm{e}}$ et au début du XX⿳亠丷厂 siècles. La variante banhado, en occupant l'espace du Paraná Tradicional et se prolongeant vers le Nord et le Nord-Ouest, montre l'influence des dialectes du Sud. Il faut rappeler que les données ont été recueillies auprès des informateurs qui habitaient à la campagne, ce qui justifie la résistance des formes plus conservatrices. 


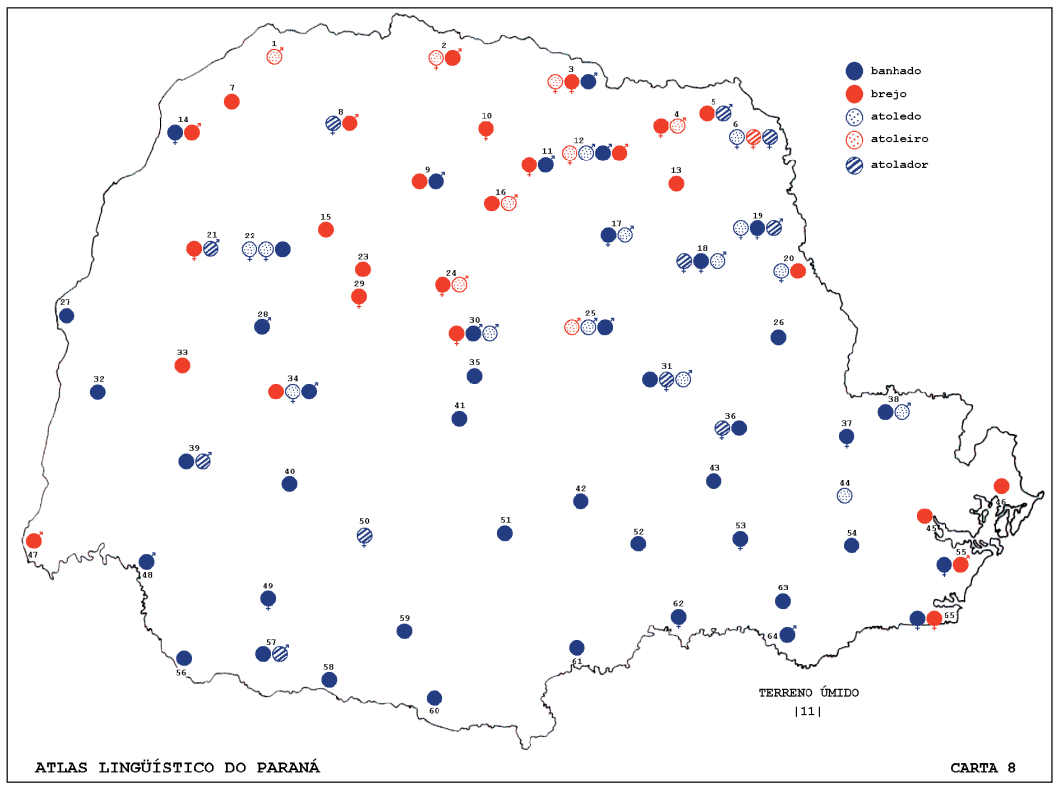

Figure 3. - Carte 8 do ALPR.

Source : Atlas Lingüístico do Paraná (Aguilera, 1994).

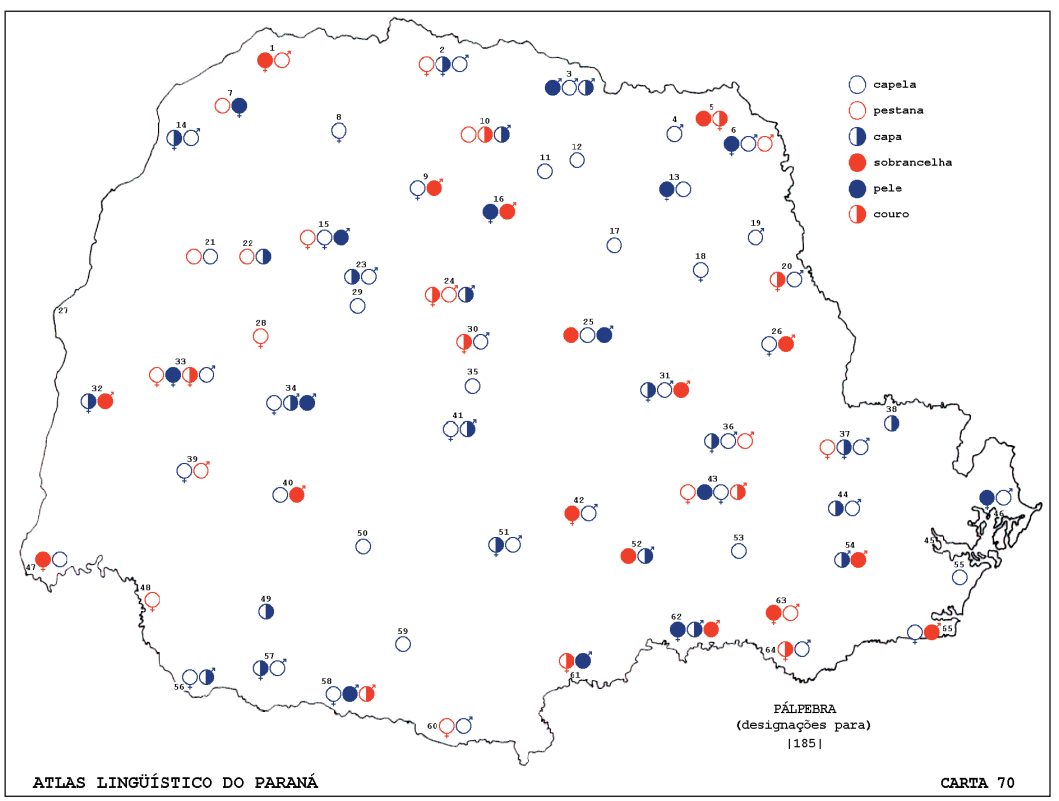

Figure 4. - Distribution des variantes lexicales pour le référent paupière : carte 70 de l'ALPR.

Source : Atlas Lingüístico do Paraná (Aguilera, 1994). 
La carte montre que parmi les variantes lexicales, au moins chez les informateurs ruraux, à la fin des années 1980, la forme culte pour paupière ne faisait pas partie du vocabulaire actif de presque tous les locuteurs; les notes de la carte 70 montrent que seulement trois informateurs connaissent le mot paupière : les deux informateurs du point d'enquête 27 (Guairá) et la locutrice de Foz do Iguaçu. La variante capela est la plus fréquente, avec 60 occurrences, et elle rayonne uniformément sur toutes les régions de l'État, aussi bien dans la zone du Paraná Moderno que Tradicional. Quant au sexe, les données indiquent l'occurrence la plus élevée dans le discours des hommes, avec 34 relevés sur $60(56,7 \%)$.

Aguilera et Silva (2015, inédit), dans une étude récente des noms des différentes parties des yeux, ont comparé les cartes de l'ALPR avec celles de l'Atlas Lingüístico da Galiza et de l'Atlas Lingüístico do Portugal e da Galíza, et elles ont constaté que capela/capela dos olhos est une forme parfaitement attestée dans les deux régions, ce qui a conduit les auteurs à affirmer que cette forme a été traitée du galicien jusqu'au portugais brésilien.

Une autre remarque des mêmes auteurs concerne le recul de capela dans le Centre-Est du domaine portugais européen dans les années 1970, en suivant à la distribution que capela a occupée dans la langue galicienne dans les années 1930. La propagation par l'intérieur du pays représente un parler plus rural qui s'est réfléchi au portugais brésilien, en particulier dans le parler du Paraná. Les auteurs ont constaté également que l'absence d'un nom spécifique ou scientifique pour une partie des yeux, comme la paupière, peut donner lieu soit à l'emploi d'un nom générique comme capa (couverture), pele (peau), couro (cuir), soit d'un nom pouvant désigner d'autres parties de la zone des yeux, comme pestana (cil) et sobrancelha (sourcil). Il faut rappeler aussi que ce processus métonymique semble être une tendance typologique ancienne, courante dans de nombreuses langues, y compris entre les langues non apparentées. Le phénomène peut être observé, en particulier, dans le cas des parties du corps humain et il affecte généralement certaines zones où des référents sont situés très près l'un de l'autre et dont les traits spécifiques sont à peine perçus par certains informateurs. C'est justement le cas de noms de parties des yeux. Concernant les phénomènes phonétiques qui distinguent le Paraná par rapport à d'autres régions du Brésil, la figure 5 présente la distribution des variantes phonétiques de la désignation borboleta (papillon). Le choix de ce référent avait des motivations diverses : un intérêt lexical d'abord dans le but de relever d'autres noms de l'insecte; en même temps, un intérêt phonétique dans la mesure où le mot permettait d'étudier les variantes des voyelles /o/ inaccentuées, dans des syllabes adjacentes, et celles $\mathrm{du} / \mathrm{r} / \mathrm{en}$ finale de syllabe interne. Les résultats sont présentés dans l'ordre. 


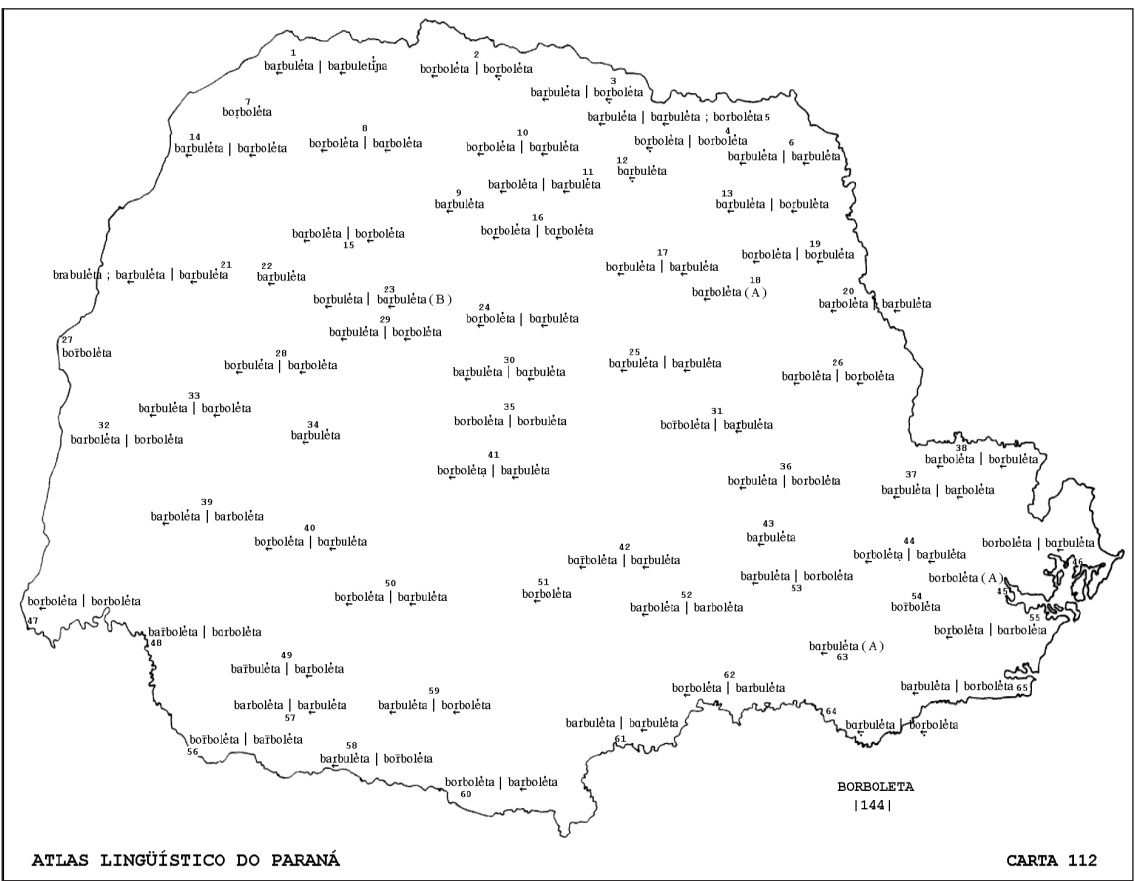

Figure 5. - Variantes phonétiques de borboleta : carte 112 de l'ALPR.

Source : Atlas Lingüístico do Paraná (Aguilera, 1994).

En analysant les données de la figure ci-dessus, nous avons constaté que, sur 130 données, la variante présentant la dissimilation de la voyelle de la première syllabe bor $>$ bar est la plus fréquente, avec 75 occurrences (58\%), suivie de la forme standard bor avec 55 occurrences (42\%). Les données indiquent également que la variable sexe n'est pertinente ni pour caractériser la variante populaire, rurale, ni la variante urbaine, cultivée. Sur les 75 occurrences de bar, $49 \%$ sont des femmes et $51 \%$ des hommes; la situation est inversée lorsqu'on compare les 55 occurrences de la variante standard montrant que $51 \%$ figurent dans les réponses des femmes et $49 \%$ dans celles des hommes.

Quant à la distribution diatopique, les résultats sont similaires : dans les 66 enregistrements relatifs aux points numérotés de 1 à 32 , correspondant approximativement au Paraná Moderno, la variante rurale est la plus fréquente avec 37 occurrences (56\%); parmi les 64 occurrences enregistrées dans les points 33 à 65, dans la zone du Paraná Tradicional, 45\% correspondent à la variante standard.

La réalisation de /r/ en finale de syllabe interne comporte 129 occurrences, dont 96 ont une articulation rétroflexe (connue aussi sous le nom /r/ 
caipira), 23 une articulation à un battement unique et 10 à battements multiples. La distribution aréale de la rétroflexe intéresse tout l'État $(74,4 \%)$, à l'exception des points 7 (Loanda / Nord-Ouest), 32 (Marechal Cândido Rondon / Ouest) et 35 (Manoel Ribas / Centre) dans lesquels seul le type à battement unique est attesté; et les points 27 (Guaíra / Ouest), 54 (Curitiba / Sud) et 56 (Barracão / extrême Sud-Ouest) où l'on relève uniquement la réalisation à battements multiples.

En ce qui concerne la réalisation de la variante majoritaire, son emploi est plus fréquent chez les femmes (53\%); concernant sa distribution géographique, la rétroflexe représente $57,3 \%$ des occurences dans le Paraná Moderno et 42,7\% dans le Paraná Tradicional. Cette variante semble avoir été introduite sur le territoire paranaense en trois phases distinctes : (i) d'abord, à la fin du XVII ${ }^{\mathrm{e}}$ siècle, sur la Côte et au Sud, avec l'arrivée des pionniers du village de São Vicente; (ii) par la suite, au cours des XVIII et $\mathrm{XIX}^{\mathrm{e}}$ siècles le long de la route des bouviers itinérants (tropeiros); et enfin, (iii) dans le dernier quart du XIX ${ }^{\mathrm{e}}$ siècle et au début du $\mathrm{XX}^{\mathrm{e}}$ siècle, par les mineurs et paulistas, dans la région maintenant connue comme Paraná Moderno. Le /r/ caipira a été propagé par la suite à d'autres régions, sauf dans les régions ayant connu une forte influence des Allemands, des Italiens, des Slaves (Sud) et des Hispaniques (Ouest et Sud-Ouest). D'autres phénomènes phonétiques, telle la réalisation de la voyelle moyenne prétonique, de la diphtongue ou et du $/ \mathrm{r} /$ intervocalique, peuvent être observés dans la figure 6 présentant la distribution des 124 occurrences des variantes phonétiques de besouro (coléoptère) contenue dans la carte 136 de l'ALPR (Aguilera, 1994).

La fermeture de la voyelle prétonique moyenne e $>\mathrm{i}$ (bisoro, bisorro) est dominante dans la prononciation des locuteurs dans 62 des 65 points d'enquête; dans les autres trois points, 26 (Jaguariaíva / Est), 27 (Guaíra / Ouest) et 35 (Manoel Ribas / Centre), le maintien de /e/ est exclusif; dans six localités on relève des cas de conservation de la voyelle et des cas de fermeture en /i/. Parmi les douze enregistrements de maintenance de /e/ sept se produisent dans le parler des femmes. En ce qui concerne la réalisation de la diphtongue $o u$, la monophtongaison est catégorique dans tous les points d'enquête. Nous avons enregistré un seul cas de conservation de la diphtongue dans la prononciation des femmes, au point 26 (Jaguariaíva / Est).

En langue portugaise, dans le langage cultivé, le /r/ intervocalique se réalise comme une constrictive à battement unique. La carte 136 de l'ALPR montre cependant que, dans la prononciation de besouro, il y a trois variantes : une constrictive alvéolaire à un seul battement, une constrictive alvéolaire à battements mutiples (vibrante) et une constrictive vélaire ou glottale; ces dernières sont les plus fréquentes, avec 47 occurrences $(37,9 \%)$ 
et plus ou moins la même répartition entre les hommes et les femmes interrogés. Cette évolution en fait un trait phonétique caractéristique du Paraná Moderno dans la mesure où il est présent dans le parler de 44 informateurs, soit 93,6\% des localités numérotées de 1 à 32. En deuxième position, concernant la fréquence d'utilisation, figure la vibrante alvéolaire multiple figurant dans 44 enregistrements $(35,5 \%)$ : elle caractérise le Paraná Tradicional, avec 77,3\% d'occurrences relevées dans les points 33 à 65 . La troisième variante, le /r/ à un seul battement, avec 33 occurrences $(26,6 \%)$, est attestée surtout dans le Paraná Tradicional, dans les régions caractérisées par une influence significative des descendants d'immigrés allemands, italiens et slaves établis majoritairement dans les régions Sud et CentreSud de l'État. On ne remarque pas de variations diasexuelles pour les trois réalisations qui présentent une répartition équilibrée dans le langage des informateurs masculin et féminin.

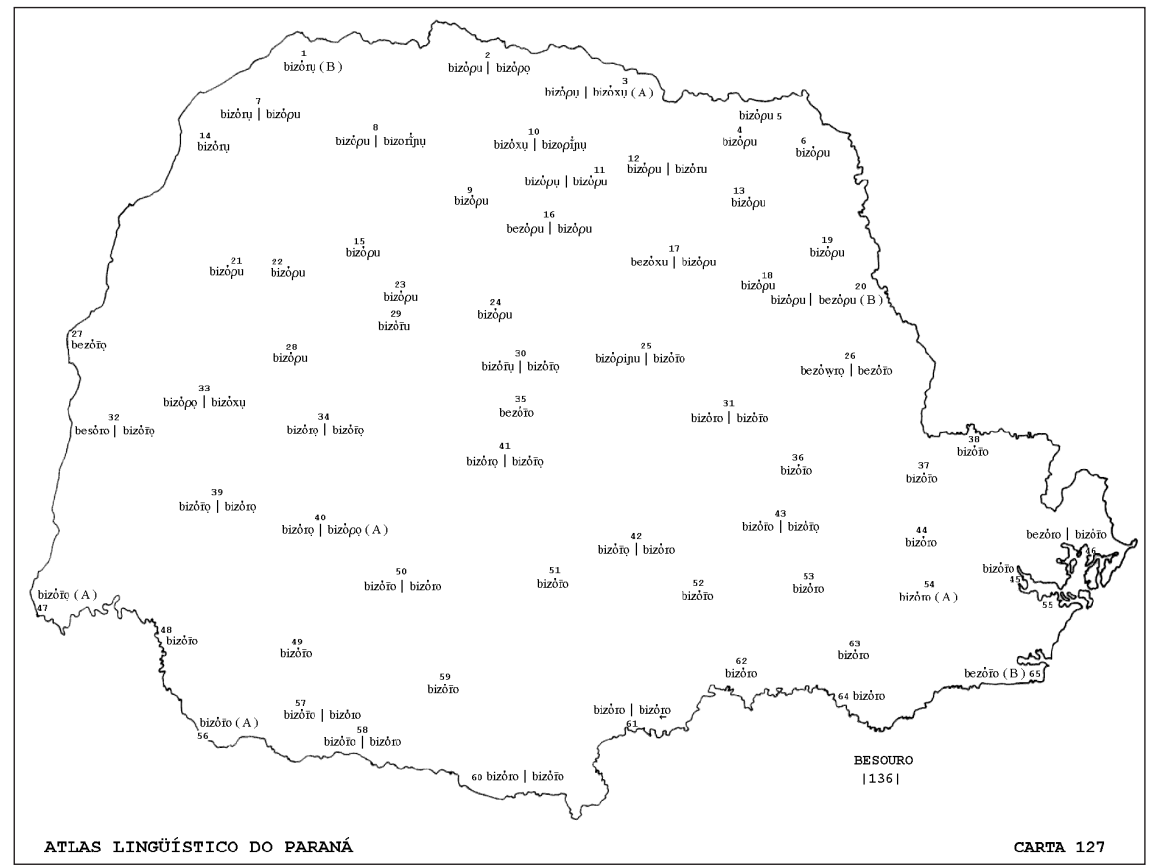

Figure 6. - Variantes phonétiques de besouro (coléoptère) : carte 136 de l'ALPR.

Source : Atlas Lingüístico do Paraná (Aguilera, 1994).

Enfin, avec la figure ci-dessous, nous présentons la distribution aréale des deux désignations corvo (corbeau) et urubu (vautour), enregistrées comme variantes en réponse à la question «oiseau noir, semblable à un poulet noir qui se nourrit de charognes d'animaux». 


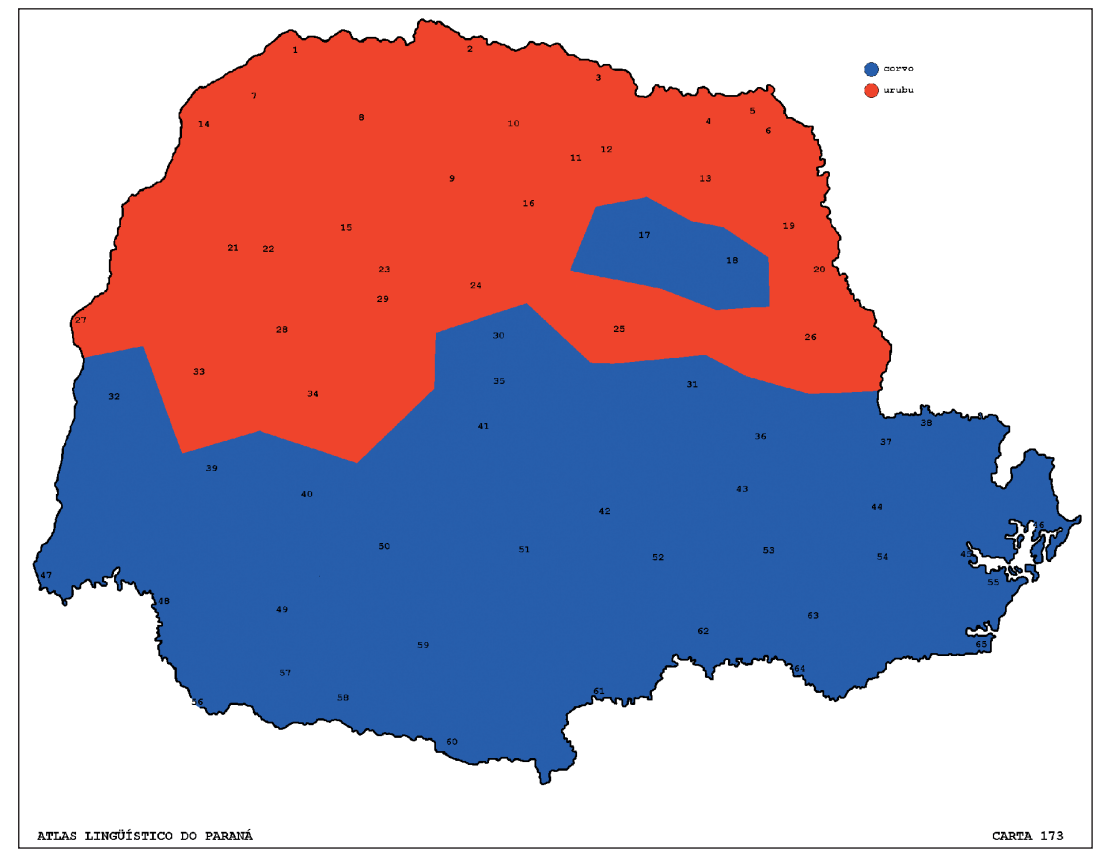

Figure 7. - Répartition des variantes isolexicales urubu /corvo : carte 173 de l'ALPR.

Source : Atlas Lingüístico do Paraná (Aguilera, 1994).

Il s'agit d'une carte synthétique construite sur la base de la carte lexicale $\mathrm{n}^{\circ} 50$. Pour sa préparation, la première réponse de chaque informateur a été prise en compte, en lui demandant tout de suite s'il connaissait un autre nom pour l'oiseau en question : systématiquement ce dernier a mentionné l'autre variante. La distribution de urubu, nom originaire du Tupi, caractérise les parlers du Paraná Moderno, où se situent les villes fondées à la fin du XIX ${ }^{\mathrm{e}}$ siècle et au début du $\mathrm{XX}^{\mathrm{e}}$ siècle; l'aire de corbeau, nom d'origine latine apporté par les immigrants européens, s'étend sur le Paraná Tradicional où il est relevé comme première chez les informateurs des localités enquêtées.

Avec ce petit échantillon, constitué de seulement sept des 191 cartes qui composent l'Atlas Lingüístico do Paraná, nous avons voulu montrer la diversité linguistique existant dans une région dont l'histoire est marquée par l'occupation successive, pendant plus de 300 ans, par des groupes culturels et linguistiques distincts, qui se sont superposés aux peuples autochtones qui l'habitaient sans doute depuis des millénaires. 


\section{Conclusion}

Cet article montre, succinctement, la genèse et l'approche méthodologique du cinquième atlas de l'État brésilien. Il présente également quelques cartes lexicales, phonétiques et une carte d'isolexiques, montrant que l'existence et la diffusion aréale des variantes sont toujours associées à la présence de l'homme et à ses incessants déplacements, en faisant l'histoire du topos et de la parole reflétés dans la continue variation linguistique : ce sont des formes parmi les plus anciennes héritées des populations autochtones et des étrangers conservés dans le langage des personnes les plus âgées, ou des variantes qui se sont mélangées à de nouveaux groupes avec lesquels les parlants sont en contact. Il s'agit aussi des formes novatrices qui font de l'ensemble des parlers paranaenses une mosaïque géante qui reflète l'histoire des occupations successives du territoire qui constituent aujourd'hui l'État de Paraná.

\section{RÉFÉRENCES BIBLIOGRAPHIQUES}

Agullera Vanderci de Andrade, 1987, Aspectos Lingüísticos da fala londrinense: esboço de um Atlas Lingüístico de Londrina, Londrina, CONCITEC-UEL/ Prefeitura Municipal de Londrina.

Aguilera Vanderci de Andrade, 1994, Atlas Lingüístico do Paraná, Curitiba, Imprensa Oficial do Estado.

Aguilera Vanderci de Andrade \& Silva Hélen Cristina da, 2015, «Convergências lexicais: a área dos olhos no Atlas Lingüístico Galego e no Atlas Lingüístico do Paraná», Estudos da Língua Galega (ELG), vol. VII.

AndRade Muricy José Cândido de, 1938, «Algumas vozes regionais do Extremo Oeste», dans Anais do primeiro Congresso da lingua nacional cantada, São Paulo, Coleção do Departamento de Cultura da Municipalidade de São Paulo.

Aragão Maria do Socorro Silva de \& Menezes Cleusa P. B. de, 1984, Atlas Linguístico da Paraíba (ALPB), Brasilia, Universidade Federal da Paraíba/CNPq, 2 vol.

Atlas Lingüístico-Etnográfico de Portugal e da Galiza, projet en cours depuis l'année 1970. Lisbonne, Centro de Linguística da Universidade de Lisboa.

Atlas Lingüístico Galego, vol. 5, Universidade de Santiago de Compostela, Instituto de Língua Galega, 1990, 1995, 1999, 2003, 2005.

Cardoso Jayme Antônio \& Westphalen Cecília Maria, 1986, Atlas Histórico do Paraná ( $2^{e}$ éd.), Curitiba, Livraria do Chaim.

CAruso Pedro, 1983, Atlas Lingüístico do Estado de São Paulo: questionário, Assis, Instituto de Letras, História e Psicologia/UNESP, Prefeitura Municipal de Assis. Disponible sur <www.ibge.gov.br/home/estatistica/populacao/estimativa 2014/estimativa_dou.shtm> [dernier accès le 18 avril 2015]. 
Ferreira Carlota da S. et al., 1987, Atlas Lingüístico de Sergipe, Salvador, Instituto de Letras/Fundação Estadual de Cultura de Sergipe.

Mercer José Luiz da Veiga, 1979, Le lexique technique des pêcheurs de Guaraqueçaba (Brésil), thèse de doctorat, Université Toulouse 2 - Le Mirail.

Muricy José Cândido, 1975, Viagem ao país dos jesuítas, Curitiba, Imprensa Oficial do Estado do Paraná.

Nascentes Antenor, 1958, Bases para a elaboração do Atlas Lingüístico do Brasil, vol. I, Rio de Janeiro, Casa de Rui Barbosa.

Nascentes Antenor, 1961, Bases para a elaboração do Atlas Lingüístico do Brasil, vol. II, Rio de Janeiro, Casa de Rui Barbosa.

Ribeiro José, Zàgari Mário Roberto Lobuglio, Passini José \& Gaio Antônio, 1977, Esboço de um Atlas Lingüístico de Minas Gerais (EALMG), vol. 1, Rio de Janeiro, Fundação Casa de Rui Barbosa; Juiz de Fora, Universidade Federal de Juiz de Fora.

Rossi Nelson, Ferreira Carlota da S. \& Isensee Dinah, 1963, Atlas Prévio dos Falares Baianos (APFB), Rio de Janeiro, INL/MEC.

Silva Neto Serafim da, 1957, Guia para estudos dialetológicos (2éd), Rio de Janeiro, INL. Disponible sur <www.google.com.br/search?q=Mapa+das+rotas +tropeiras+no+sul+do+Brasil $>$ [dernier accès le 23 avril 2015]. 